\title{
DESARROLLO DE LAS COMPETENCIAS DIGITALES EN LOS DOCENTES UNIVERSITARIOS EN TIEMPO PANDEMIA: UNA REVISIÓN SISTEMÁTICA
}

\author{
DEVELOPMENT OF THE DIGITAL COMPETENCES IN \\ THE UNIVERSITY TEACHERS IN PANDEMIC TIME: \\ SYSTEMATIC REVIEW
}

\author{
Mónica G. Rambay Tobar ${ }^{1}$, \\ Juan De La Cruz Lozado
}

\section{RESUMEN}

El presente artículo tiene como objetivo analizar en las evidencias científicas el desarrollo de las Competencias Digitales en los Docente Universitario, quienes se vieron obligados a replantear sus programas de estudios en un entorno virtual debido a la pandemia mundial de la COVID-19. La búsqueda se realizó utilizando las bases de datos científicas Scopus, ProQuest, Ebsco, así como la aplicación web Mendeley. Se incluyeron artículos que identificaban las competencias digitales en docentes universitarios en el presente año. Se excluyeron estudios realizados en instituciones de educación primaria y secundaria. Como conclusiones se establecen que será necesario elaborar un plan de fortalecimiento para las competencias docentes que no se han desarrollado satisfactoriamente, especialmente las relacionadas con el rol del docente (planificación, desarrollo, conducción de experiencias de aprendizaje presenciales con TIC), la creación del contenido digital, y la seguridad.

PALABRAS CLAVE: competencias digitales, docentes universitarios, entornos virtuales

1 Licenciada en Supervisión y Administración Educativa Bilingüe, Magister en Dirección del Talento Humano, Universidad Espíritu Santo, Ecuador. E-mail mrambay@ecotec.edu.ec.

2 Magister en Didáctica Universitaria e Investigación Educativa, Doctorado en Gestión y Ciencias de la Educación.E-mail: dlozadoj@ucvvirtual.edu.pe 


\begin{abstract}
The objective of this article is to analyze in the scientific evidences the development of Digital Competences in University Teachers, who were forced to rethink their study programs in a virtual environment due to the global pandemic of COVID-19. The search was carried out using the scientific databases Scopus, ProQuest, Ebsco, as well as the Mendeley web application. Articles were included that identified digital competences in university teachers this year. Studies conducted in institutions of primary and secondary education were excluded. As conclusions are established that it will be necessary to develop a strengthening plan for teaching competences that have not been developed satisfactorily, especially those related to the teacher's role (planning, development, conducting face-to-face learning experiences with ICT), creation digital content, and security.
\end{abstract}

KEY WORDS: digital competences, university teachers, virtual environment

\title{
INTRODUCCIÓN
}

El tema de investigación que se aborda se planteó a partir de las circunstancias de la pandemia ocasionada por el COVID 19. El 16 de marzo del año 2020, el presidente de la República Lenín Moreno Garcés, declaró el Estado de Excepción en todo el territorio ecuatoriano, con el propósito de contener la transmisión del coronavirus (Secretaría General de Comunicación de la Presidencia, 2020).

En este marco, el Consejo de Educación Superior (CES) el 25 de marzo de 2020 estableció una normativa con medidas excepcionales ante el estado de emergencia sanitaria que atraviesa el Ecuador, a fin de garantizar los derechos de todos los actores que forman parte del Sistema de Educación Superior. Asimismo, se autorizó que las universidades ajusten las actividades de aprendizaje para que puedan ser desarrolladas e impartidas mediante el uso de tecnologías interactivas multimedia y entornos virtuales de aprendizaje, a través de plataformas digitales. Del mismo modo, las IES debían garantizar que estos recursos de aprendizaje estén disponibles para todos los estudiantes y personal académico.

Estos cambios en la sociedad obligaron a que las universidades introduzcan nuevos modelos educativos; y que, tanto docentes como estudiantes, adquieran las nuevas competencias del siglo XXI: creatividad, trabajo colaborativo y alfabetización digital. Los entornos virtuales, las herramientas tecnológicas, y las aplicaciones con enfoque académico, se han convertido en medios para desarrollar dichas competencias. 
La dura realidad de la pandemia del coronavirus y sus consecuencias económicas, afectó a muchas empresas, incluyendo la educativa. Unas optaron por cerrar, pero otras replantearon sus líneas de ventas y estrategias para sobrevivir a la emergencia sanitaria, tratando de frenar la exponencial curva de contagios para precautelar su salud y la de sus familias. Sin embargo, las instituciones de educación superior se han mantenido activas con el trabajo coordinado de sus directivos, administrativos y docentes, para seguir cumpliendo con la gran responsabilidad social de formación académica y profesionalización de sus estudiantes.

García-Peñalvo, Corell, Abella-García, \& Grande (2020) manifiestan que debido a esta inesperada pandemia no hubo tiempo para rediseñar los programas de las asignaturas que se pasaron a dictar en la modalidad online, sin la certeza de que ni los docentes ni los estudiantes contaban con los recursos tecnológicos, las competencias digitales y una buena actitud frente al cambio. En este contexto, se han evidenciado cuatro grandes brechas relacionadas a: 1) acceso a los dispositivos electrónicos y conexión de internet; 2) tiempo y calidad del uso de internet, ya que muchos hogares sí cuentan con los dispositivos pero este es compartido por varios integrantes de la familia; 3) competencias digitales de los docentes y estudiantes; y 4) evaluación online, que se va a desarrollar en un contexto inseguro en lo que se refiere a la protección de datos y de derechos de privacidad de los docentes y estudiantes, asimismo de los riesgos a los que están expuestos los sistemas que sustentan la docencia y la evaluación online (Fernández Enguita, 2020).

En la "Conferencia Mundial sobre la Educación Superior", convocada por la UNESCO en el 2001, Francisco López Segrera ya predecía que las nuevas tecnologías de la información y comunicación (TIC) estaban introduciendo un nuevo paradigma en el proceso enseñanza - aprendizaje, el cual introduce otros conceptos de "colaboración", "enseñanza asincrónica". Además, se hicieron recomendaciones de cómo afrontar los desafíos más urgentes. Como por ejemplo: la actualización permanente de los docentes, de los contenidos y del currículo; la introducción de redes electrónicas para el aprendizaje; traducción y adaptación de las principales contribuciones científicas; modernización de los sistemas de gestión y dirección; e integración y complementación de la educación pública y privada así como de la educación formal, informal y a distancia (López Segrera, 2008).

David Ausubel definió el aprendizaje significativo como un proceso de relación entre conocimiento previo y la propia experiencia con la nueva información que adquiere el estudiante. Este aprendizaje es importante en la educación por- 
que es el elemento humano por excelencia que permite adquirir y almacenar una extensa cantidad de ideas e información, representada por cualquier campo del conocimiento (Anaya Velasco, Díaz Rodríguez, \& Martínez Hernández, 2012).

El aprendizaje significativo se promueve con el uso de las TIC; el docente deberá adaptar o crear los recursos didácticos y planificar actividades que capten la atención y el interés de los estudiantes, creando un ambiente de inmersión donde predomine la interacción, y el docente se convierta en un guía en el proceso cognitivo. Para lograr el uso correcto y seguro de las TIC, hay que enfocarse en el desarrollo de las competencias digitales en los docentes, que enmarcadas en el ámbito educativo establezcan una relación apropiada del uso de TIC, junto con el desarrollo de competencias didácticas y metodológicas que integren su uso en la educación y la tecnología (Zavala, Muñoz, \& Lozano, 2016).

En publicaciones recientes, el término competencia no solo se refiere a las habilidades, lo que refleja la necesidad de un contenido más amplio y profundo de los conceptos. La relación entre competencia y habilidades se define en un proyecto de la OCDE de la siguiente manera: "Una competencia es más que solo conocimiento y habilidades. Implica la capacidad de satisfacer demandas complejas, aprovechando y movilizando recursos psicosociales (incluidas habilidades y actitudes) en un contexto particular" (Organisation for Economic Cooperation and Development, 2005).

La OCDE lanzó un proyecto "Definición y selección de competencias del programa de la OCDE" (2005) que identifica las competencias clave para una vida exitosa y una sociedad que funcione bien, y las clasifica en tres categorías amplias:

1) Utilización de herramientas de forma interactiva,

2) Interacción en grupos heterogéneos, y

3) Actuación de forma autónoma.

Cada una de estas competencias clave implica la unión de conocimientos, cognitivos y habilidades prácticas, así como componentes sociales y de comportamiento que incluyen actitudes, emociones, valores y motivaciones. La parte subyacente del marco es el pensamiento y la acción reflexiva. El pensamiento reflexivo exige procesos mentales relativamente complejos e implica el uso de habilidades metacognitivas, habilidades creativas, y adoptar una postura crítica. 
La primera competencia clave, utilización de herramientas de forma interactiva, está cerca de las competencias digitales. Significa la capacidad de utilizar la tecnología con otras personas para comunicarse, trabajar, jugar, etc. Una persona debe tener la capacidad de aprovechar el potencial de las TIC para transferir la forma de trabajar, acceder a la información e interactuar con otros (Ilomäki, Kantosalo, \& Lakkala, 2017).

La competencia digital es una competencia clave, y se refiere al uso seguro y crítico de la gama completa de las tecnologías digitales para la información, la comunicación y la resolución de problemas básicos en todos los aspectos de la vida. Asimismo es importante considerar que siendo una competencia transversal, la competencia digital también ayuda a dominar otras competencias claves, como la comunicación, las habilidades lingüísticas o las habilidades básicas en matemáticas y ciencias", tal como lo señala Riina Vuorikari (2015) en su artículo "Becoming digitally competent: A task for the 21st-century citizen".

Durante los últimos años, la competencia digital se ha convertido en un concepto clave en la discusión de qué tipo de habilidades y comprensión deben tener las personas en la sociedad del conocimiento. Para comprender mejor la naturaleza de la competencia digital, la Comisión Europea ha desarrollado el Marco Europeo de Competencias Digitales para los Ciudadanos (2017), con un total de 21 competencias que se dividen en cinco áreas: 1) alfabetización en información y datos; 2) comunicación y colaboración; 3) creación de contenido digital;4) la seguridad; y 5) resolución de problemas.

Área 1: Alfabetización en Información y Datos: Navegación, búsqueda y filtrado de datos, información y contenido digital. Evaluación de datos, información y contenido digital. Gestión de datos, información y contenido digital

Área 2: Comunicación y Colaboración: Interactuar a través de tecnologías digitales. Compartir a través de tecnologías digitales. Compromiso con la ciudadanía a través de tecnologías digitales. Colaboración a través de tecnologías digitales. Netiqueta. Gestionar la identidad digital.

Área 3: Creación de Contenido Digital: Desarrollar el contenido digital. Integrar y reelaborar contenidos digitales. Copyright y licencias. Programación.

Área 4: Seguridad: Protección de dispositivos. Protección de datos personales y privacidad. Protección de la salud y el bienestar. Protección del medio ambiente. 
Área 5: Resolución de Problemas: Resolver problemas técnicos. Identificación de necesidades y respuestas tecnológicas. Uso creativo de tecnologías digitales. Identificar brechas de competencia digital.

Para Sandi-Delgado \& Sanz (2020) el concepto de competencias digitales es relativamente nuevo, describe las habilidades relacionadas con la tecnología, y varía según la importancia que la institución o el investigador le brinde. En los últimos años, se han utilizado varios términos para describir las habilidades y competencias de utilizar tecnologías, tales como: competencias en TIC, competencias tecnológicas, competencias en tecnología de la información, alfabetización en información, alfabetización digital y habilidades digitales. Las competencias abarcan diferentes unidades cognitivas, las cuales se fundamentan en habilidades pedagógicas y tecnológicas; y componentes afectivos: conocimientos, valores, ética, actitudes; apertura, recepción, persistencia y aprender a partir de errores. La alfabetización digital del docente está relacionada con el uso correcto y crítico de las TIC en la educación con el objetivo de proponer soluciones a problemas cognitivos; a su vez, el docente se convierte en un mediador entre la tecnología y los procesos formativos. Se cree ampliamente que el desarrollo de la competencia digital debe comenzar a una edad temprana, pero las decisiones sobre los tipos de tecnologías y la cantidad de tiempo dedicado a ellas deben considerarse cuidadosamente.

Sandí-Delgado (2020) define a las competencias tecnológicas como un conjunto de saberes relacionados con el uso y dominio de las tecnologías digitales y otras áreas del conocimiento relacionadas, que permiten desarrollar un comportamiento propio ante un evento con el fin de potenciar el mejoramiento personal y/o profesional.

Según una investigación realizada por Rangel y Peñalosa (2013), no existe un acuerdo sobre las competencias digitales en los docentes universitarios, por lo que asumen los siguientes indicadores: (a) Procesos que incluyen el empleo de las TICs, en donde el docente universitario deberá desarrollar ciertas destrezas en técnicas informáticas, actualización profesional constante, metodología docente acorde a los desafíos y actitud hacia las nuevas tecnologías; (b) Dimensiones de rasgos formativos que abarquen aspectos, tales como el manejo instrumental, capacidad cognitiva, el factor actitudinal hacia las nuevas tecnologías y el afianzamiento axiológico acorde con todo lo anterior; (c) Un acercamiento hacia los mecanismos relacionados con la gestión del conocimiento que se enfoquen en aspectos esenciales, tales como el manejo de nociones básicas sobre las TICs, 
acrecentar el nivel de conocimiento sobre las nuevas tecnologías y por último la implementación de mecanismos de generación del conocimiento. Según Levano-Francia y otros (2019) las competencias digitales no pueden desvincularse de la alfabetización digital, ya que los docentes universitarios deben desarrollar una serie de competencias que les permitan el manejo correcto de los recursos tecnológicos, pedagógicos, informacionales, comunicativos y axiológicos.

La generación a la que pertenecen los docentes universitarios no debe influenciar en la importancia de su capacitación en las TICs; es decir, que inclusive los docentes de la última generación o "nativos digitales" (generación "Z"). Las instituciones de educación superior deben incitar a la alfabetización digital de sus docentes, a través de certificaciones en competencias digitales que les permita tener el know-how para implementar con seguridad, entusiasmo y motivación estrategias creativas e innovadoras en sus contextos educativos, además de incentivar a los estudiantes para que conozcan los beneficios y potencialidades pedagógicas de las TIC (Amaya-Amaya, Zúñiga-Mieles, Salazar-Blanco, \& Ávila-Ramírez, 2018).

Este artículo tiene como objetivo identificar las evidencias científicas que explican el desarrollo de las competencias digitales en los docentes universitarios.

\section{MÉTODO}

El presente artículo de revisión sistemática se elaboró incluyendo estudios que involucraban a estudiantes, docentes, y directivos de instituciones de educación superior. Los estudios que incluían las competencias digitales en docentes universitarios se consideraron criterios de elegibilidad.

Debido al limitado número de estudios sobre las competencias digitales en docentes universitarios durante la pandemia del COVID-19, el principal objetivo de este artículo es identificar las evidencias científicas que explican el desarrollo de las competencias digitales en los docentes universitarios.

Las búsquedas se realizaron utilizando títulos que identificaban las competencias digitales en docentes universitarios en las siguientes bases de datos científicas: Scopus, ProQuest, Ebsco, así como la aplicación web Mendeley. Las búsquedas se realizaron a partir del 18 de octubre de 2020, y se excluyeron estudios realizados en instituciones de educación primaria y secundaria.

El proceso de selección de estudios fue realizado en dos instancias. La primera, consistió en revisar los títulos y resúmenes de las referencias encontradas con 
la estrategia de búsqueda. Se distinguieron los estudios potencialmente elegibles. La segunda, radicó en revisar el texto completo de los artículos preseleccionados para confirmar la elegibilidad.

De los cuatro estudios incluidos en esta revisión, uno de ellos fue publicado en el 2018, mientras que los otros tres fueron publicados entre mayo y agosto de 2020. Coincidentemente, estos cuatro artículos son de tipo descriptivos, y tienen su origen en países americanos: Argentina, Colombia, y México (2).

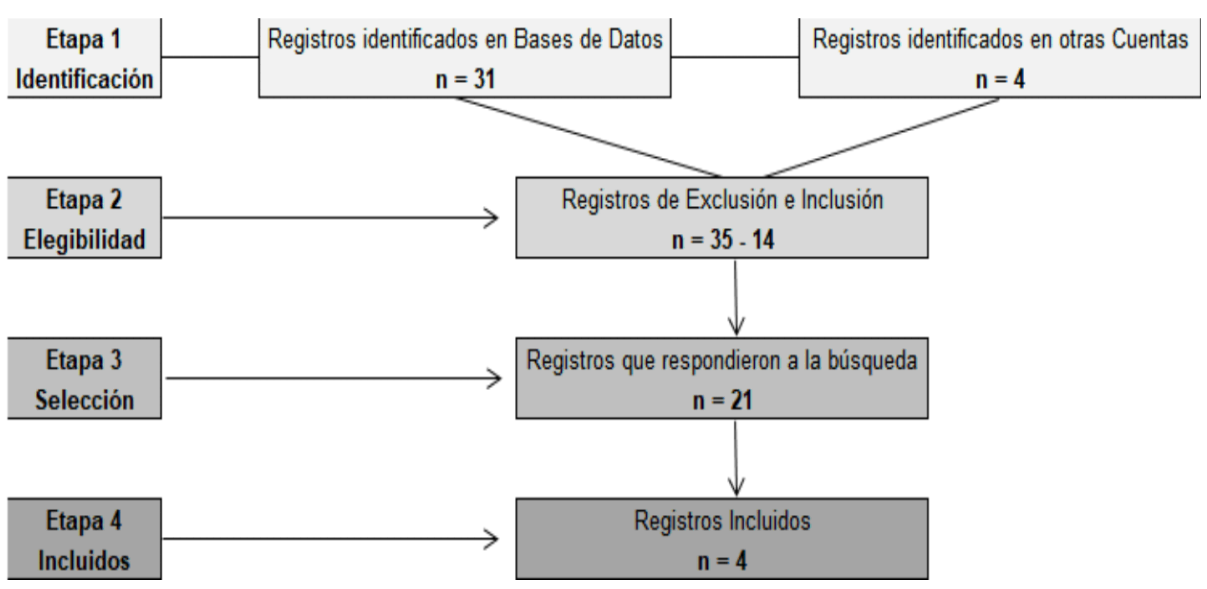

Figura 1. Flujograma de las etapas de criterios de elegibilidad

(de inclusión y exclusión)

\section{RESULTADOS}

Las estrategias de búsqueda proyectaron 35 referencias. En la primera instancia del proceso de selección, se eliminaron 14 referencias que no cumplían los criterios de inclusión al revisar el título y el resumen. En la segunda instancia, se leyeron las 21 referencias seleccionadas para confirmar la elegibilidad. De la revisión de estas 21 referencias de texto completo seleccionadas se excluyeron 17 estudios que no cumplieron con nuestros criterios de inclusión.

Los resultados de los 4 estudios escogidos para la elaboración de este artículo, se presentan a continuación de forma descriptiva. 
Tabla 1

DESCRIPCIÓN DE LOS ESTUDIOS INCLUIDOS

\begin{tabular}{|c|c|c|c|c|c|c|c|c|c|}
\hline $\mathbf{N}$ & $\begin{array}{l}\text { Titulo del } \\
\text { documento }\end{array}$ & Autores & Año & $\begin{array}{l}\text { Tipo de } \\
\text { artículo }\end{array}$ & $\begin{array}{c}\text { Contexto } \\
\text { del estudio }\end{array}$ & Revista & Indexación & Comentario & Link o DOI \\
\hline 1. & $\begin{array}{l}\text { Competencias } \\
\text { Digitales en Docentes } \\
\text { de Eduoación } \\
\text { Supetiot: Niveles de } \\
\text { Dominioy } \\
\text { Necesidades } \\
\text { Formativas }\end{array}$ & 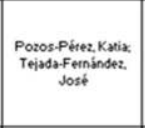 & 2018 & $\begin{array}{l}\text { Artículo de } \\
\text { Investigación }\end{array}$ & México & $\begin{array}{l}\text { Revista Digital } \\
\text { de } \\
\text { Investigación } \\
\text { en Docencia } \\
\text { Universitaria }\end{array}$ & Soielo & $\begin{array}{l}\text { Los resultados indican un dominio } \\
\text { medio bajo en las competencias } \\
\text { digitales que cortesponden con el rol } \\
\text { de docencia (planificación desartollo } \\
\text { y conduoción de enpertiencias de } \\
\text { aprendizaje gla evaluación con apogo } \\
\text { de las TiC). }\end{array}$ & $\begin{array}{l}\text { dok } \\
\text { hetp.lldedoiorgh0.19083hidu. } 12.558\end{array}$ \\
\hline 2. & $\begin{array}{l}\text { Virtualidady } \\
\text { Eduosción en } \\
\text { Tiempos de Covid.19. } \\
\text { Un Estudio Empirioo } \\
\text { en Argentina }\end{array}$ & $\begin{array}{l}\text { Expósino, Cristian } \\
\text { David: Marsollet, } \\
\text { Rounana Graciela }\end{array}$ & 2020 & $\begin{array}{l}\text { Artículo de } \\
\text { investigación }\end{array}$ & Argentins & $\begin{array}{l}\text { Eduoacilióny } \\
\text { Humanismo }\end{array}$ & Disinet & 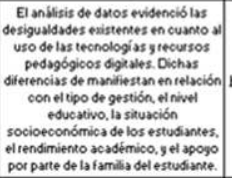 & 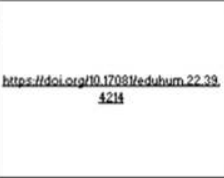 \\
\hline 3. & $\begin{array}{l}\text { Competencias } \\
\text { Digitales Docentes yel } \\
\text { Reto de la Educación } \\
\text { Virtual derivado de la } \\
\text { CoviD-19 }\end{array}$ & $\begin{array}{l}\text { Martínez-Garcés. } \\
\text { Josnet, Garcís. } \\
\text { Fuenmagor. } \\
\text { Jacqueline }\end{array}$ & 2020 & $\begin{array}{l}\text { Artículo de } \\
\text { Investigación }\end{array}$ & Colombia & $\begin{array}{l}\text { Educación y } \\
\text { Humanismo }\end{array}$ & Dialnet & 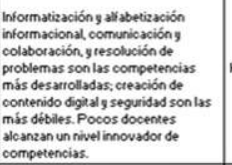 & $\begin{array}{l}\text { hetps s:ldoiorghto.17081/eduhum 22.39. } \\
4114\end{array}$ \\
\hline 4. & $\begin{array}{l}\text { Retos Educativos } \\
\text { Durante is Pandemis } \\
\text { de COVID.19. Una } \\
\text { Encuesta } \\
\text { Profesores de la } \\
\text { UNAM }\end{array}$ & 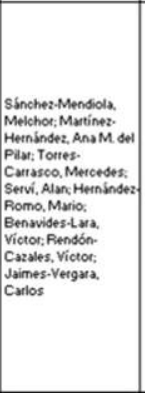 & 2020 & $\begin{array}{l}\text { Artículo de } \\
\text { Investigación }\end{array}$ & Mexrico & $\begin{array}{c}\text { Revista Digital } \\
\text { Universitaria }\end{array}$ & Latindex & 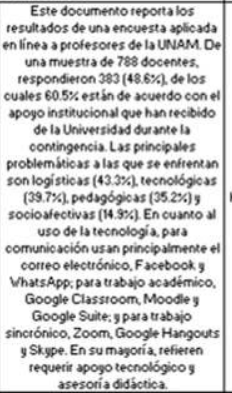 & 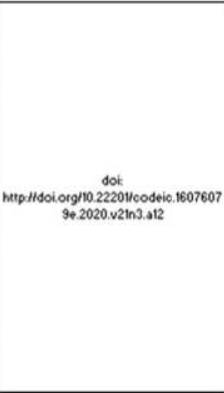 \\
\hline
\end{tabular}

Fuente: Elaboración propia

En el primer estudio, participaron 247 docentes de 20 instituciones universitarias de la Zona Metropolitana del Valle de México. Según Pozos-Pérez y TejadaFernández (2018), el docente universitario debe desarrollar siete competencias digitales: 1) Planificación y diseño de experiencias de aprendizaje en ambientes presenciales y virtuales; 2) Desarrollo y conducción de experiencias de aprendizaje colaborativas presenciales y en red; 3) Orientación, guía y evaluación de los procesos de construcción del conocimiento en entornos presenciales y virtuales; 4) Gestión del conocimiento y desarrollo profesional con apoyo de las TIC; 5) Investigación, desarrollo e innovación pedagógica con/para el uso de las TIC en educación; 6) Diversidad, ética y uso responsable de las TIC en el desempeño profesional docente; 7) Medio ambiente, salud y seguridad laboral con el uso de las TIC en la profesión docente. 
Las competencias digitales que más dominan los docentes de este estudio están relacionadas con el compromiso y la responsabilidad social con el uso de las TIC (6 y 7); mientras que las competencias menos desarrolladas (1, 2 y 3 ) corresponden al rol de la docencia y están relacionadas con la planificación, así como el desarrollo y conducción de experiencias de aprendizaje presenciales con TIC. Finalmente, las competencias que tienen un nivel de dominio medio (4 y 5) están relacionadas con la gestión, la investigación y el desarrollo profesional.

En el segundo estudio, participaron 777 sujetos entre docentes y directivos de la Provincia de Mendoza en Argentina, donde el 36,16 \% corresponde a nivel universitario. Expósito y Marsollier (2020) presentan un análisis con resultados significativos sobre las estrategias y recursos, tanto pedagógicos como tecnológicos, utilizados por los docentes en el marco del confinamiento preventivo social obligatorio como consecuencia del COVID-19.

La primera está relacionada con la variable Tipo de Gestión Institucional, e indica que las instituciones estatales presentan un mayor manejo de herramientas tecnológicas populares, tales como: WhatsApp, redes sociales, etc., mientras que las instituciones particulares o privadas utilizan tecnologías específicas; es decir, herramientas más adecuadas y complejas que necesitan un conocimiento determinado para su uso. Entre ellas se encuentran: las plataformas, las videoconferencias, los foros, los blogs o páginas web.

La segunda relación significativa tiene que ver con el nivel socioeconómico del estudiante respecto al uso de las tecnologías. Los resultados demuestran que las tecnologías específicas son utilizadas por los estratos medios, medio altos y altos; mientras que las tecnologías populares son utilizadas por los estratos bajos. Otra relación importante se produce con la variable del nivel educativo donde el docente desarrolla su actividad pedagógica. Los valores medios de los niveles superiores son los que presentan el mayor uso de las tecnologías específicas, especialmente las plataformas. En síntesis, los resultados obtenidos permiten identificar que existe una relación positiva entre el uso de los recursos pedagógicos/didácticos digitales por parte del docente.

El tercer estudio fue desarrollado en una institución de educación superior, ubicada en el Valle del Cauca - Colombia, donde participaron 52 docentes vinculados al periodo académico 2020-I. Los autores de este estudio fueron MartínezGarcés y Garcés-Fuenmayor (2020) quienes presentan los resultados de su inves- 
tigación basándose en las competencias digitales señaladas por Moll (2017): 1) Información y Alfabetización Internacional; 2) Comunicación y colaboración; 3) Creación del contenido digital; 4) Seguridad; 5) Resolución de problemas.

La primera competencia se refiere a la facilidad para localizar, identificar y clasificar la información digital; un 57,69\% de los docentes consideran que es fácil localizar en la red contenido digital relevante para sus clases, demostrando que a través de las bibliotecas y repositorios digitales satisfacen sus necesidades de información. De igual manera, un 57,69\% considera fácil y un 25\% tiene una tendencia positiva a identificar cuál del contenido localizado es más idóneo para sus clases. No obstante, llama la atención que un 13,46\% respondió negativamente a este indicador evidenciando que les resulta difícil esta acción. En cuanto a clasificar la información un 78,85\% lo considera fácil y un 15,38\% mantiene una tendencia positiva sobre ello.

En la segunda competencia existe una tendencia medianamente positiva ya que el 48,08\% de los docentes utiliza entornos digitales para compartir recursos utilizando herramientas en línea promoviendo la conexión y colaboración con otros a través de la construcción de redes de socialización. Sin embargo, un porcentaje considerable de $34,62 \%$ piensa que establecer comunicación con sus estudiantes bajo entornos digitales es definitivamente fácil contemplando el uso adecuado de los distintos formatos disponibles.

En la tercera competencia juega un papel relevante la creatividad para poder generar, editar, integrar y reelaborar contenidos digitales, considerando los aspectos de la propiedad intelectual. En esta competencia la tendencia fue positiva, ya que el 32,69\% de los docentes afirma que para ellos es fácil, junto a un $42,31 \%$ que también se alinea a este criterio al producir contenidos digitales completamente nuevos para utilizarlos en sus clases bajo entornos digitales.

La cuarta competencia, está relacionada con los conocimientos, actitudes y habilidades de los docentes para diseñar y desarrollar experiencias de aprendizaje digitalmente responsables con la debida privacidad, integridad y eficacia de la información obtenida del Internet; las respuestas se concentran en tendencias no absolutas, donde un 32\% se inclina a la facilidad, mientras que un 30,77\% considera que es difícil utilizarla. Por otra parte, la protección de datos personales muestra una alta tendencia positiva pues un 40,38\% indica que es fácil para los docentes encuestados mantenerla. Sin embargo, la protección de identidad si se mantiene con una inclinación negativa de $32,69 \%$ y un $17,31 \%$ que consideran defi- 
nitivamente difícil establecer protocolos de protección digital al momento de desarrollar sus clases bajo entornos virtuales.

Finalmente, la quinta competencia está orientada a la identificación de necesidades, toma de decisiones y resolución de problemas conceptuales a través de las herramientas digitales. Los resultados reflejan una tendencia positiva de $46,15 \%$ junto a un $34,62 \%$ que la considera absolutamente fácil, orientando asíla construcción del conocimiento desde la virtualidad. De la misma forma, en cuanto a usar de manera creativa la tecnología en sus asignaturas dictadas bajo entornos digitales, los resultados se concentran altamente positivos con un 36,54\% sumado a un $42,31 \%$ que la considera absolutamente fácil.

El cuarto estudio fue realizado en una de las universidades más grandes del mundo, la Universidad Nacional Autónoma de México (UNAM). El 25 de marzo de 2020, se envió un cuestionario de forma masiva, vía correo electrónico, a 788 docentes; de los cuales dos direcciones resultaron inválidas. Al quinto día de enviado el cuestionario, respondieron 383 docentes; es decir, el 48,6\% de la población encuestada. Entre los resultados del estudio realizado por SánchezMendiola, Martínez-Hernández, Torres-Carrasco, Agüero-Servin, HernándezRomo, Benavides-Lara, Rendón Cazares, y Jaimes-Vergara (2020), agruparon cuatro tipo de problemáticas a las que se están enfrentando los docentes para transformar sus actividades a la modalidad a distancia: 1) Logísticas; 2) Tecnológicas; 3) Pedagógicas; y 4) Socioafectivas.

El 43,3\% de los docentes considera que las problemáticas más frecuentes que tienen que enfrentar son las logísticas; las cuales están relacionadas con el manejo del tiempo, los horarios de clases, los espacios físicos para trabajar en esta modalidad, la comunicación institucional, entre otras. En segundo lugar, se encuentran las problemáticas tecnológicas, donde el 39,7\% de los docentes coinciden que se torna dificultoso el acceso a internet, la disposición de equipos de cómputo, los conocimientos de plataformas educativas, entre otros. Un tercer grupo de docentes $(35,2 \%)$ coinciden con las problemáticas pedagógicas, es decir, no tienen suficiente conocimiento de herramientas didácticas de educación a distancia, el manejo de grupos a distancia, la evaluación de estudiantes, entre otros. Finalmente, un grupo reducido de docentes (14,9\%) enfrenta las problemáticas socioafectivas; es decir, aquellas que están relacionadas con aspectos emocionales, afectivos y de salud, sentimientos de tristeza, frustración, ansiedad, cansancio, entre otras. 
Adicionalmente, este estudio obtuvo 36 respuestas abiertas que se agruparon en cuatro categorías: 1) La dinámica y carga de trabajo de la modalidad no presencial; 2) Las dificultades que los docentes perciben que tienen sus estudiantes, tales como problemas de actitud y disposición, dificultades en la disponibilidad y uso de tecnologías; 3) Las situaciones personales que les dificulta trabajar en esta modalidad; $y, 4)$ Las situaciones institucionales de las dependencias.

\section{Tabla 2}

\section{COMPETENCIAS DIGITALES IDENTIFICADAS}

\begin{tabular}{|l|l|l|l|}
\hline \multicolumn{1}{|c|}{ Universidad / Sector } & $\begin{array}{l}\text { Competencias Digitales } \\
\text { más Desarrolladas }\end{array}$ & $\begin{array}{l}\text { Competencias Digitales } \\
\text { Medianamente } \\
\text { Desarrolladas }\end{array}$ & $\begin{array}{l}\text { Competencias Digitales } \\
\text { Menos Desarrolladas }\end{array}$ \\
\hline $\begin{array}{l}\text { Universidades Zona } \\
\text { Metropolitana del Valle de } \\
\text { México }\end{array}$ & $\begin{array}{l}\text { Compromiso y } \\
\text { Responsabilidad Social } \\
\text { con el Uso de las TIC }\end{array}$ & $\begin{array}{l}\text { Gestión. } \\
\text { Investigación. } \\
\text { Desarrollo profesional. }\end{array}$ & $\begin{array}{l}\text { Rol de Docencia: } \\
\text { Planificación, Desarrollo, } \\
\text { Conducción de } \\
\text { experiencias de } \\
\text { aprendizaje presenciales } \\
\text { con TIC }\end{array}$ \\
\hline $\begin{array}{l}\text { Universidad - Ciudad de } \\
\text { Mendoza, Argentina }\end{array}$ & $\begin{array}{l}\text { Rol Docencia: } \\
\text { digitalización del material } \\
\text { didáctico, y adaptación de } \\
\text { los programas de estudio. }\end{array}$ & & \\
\hline $\begin{array}{l}\text { Universidad - Valle del } \\
\text { Cauca, Colombia }\end{array}$ & Resolución de Problemas. & $\begin{array}{l}\text { Alfabetización } \\
\text { Internacional } \\
\text { Comunicación y } \\
\text { Colaboración }\end{array}$ & $\begin{array}{l}\text { Creación del contenido } \\
\text { digital. } \\
\text { Seguridad. }\end{array}$ \\
\hline $\begin{array}{l}\text { Universidad Nacional } \\
\text { Autónoma de México }\end{array}$ & Socio afectivas & & $\begin{array}{l}\text { Logísticas } \\
\text { Tecnológicas } \\
\text { Pedagógicas }\end{array}$ \\
\hline
\end{tabular}

Fuente: Elaboración propia.

\section{DISCUSIÓN}

De acuerdo a lo señalado en la introducción de este artículo, y a partir de los resultados obtenidos en los estudios presentados, se reconoce que en el estudio desarrollado en varias universidades de la Zona Metropolitana del Valle de México, las competencias digitales que más dominan los docentes están relacionadas con el compromiso y la responsabilidad social con el uso de las TIC, mientras que las competencias menos desarrolladas corresponden al rol de la docencia y están relacionadas con la planificación, así como el desarrollo y conducción de experiencias de aprendizaje presenciales con TIC. Las competencias que tienen un nivel de dominio medio están relacionadas con la gestión, la investigación y el desarrollo profesional. 
En lo que se refiere al estudio realizado en instituciones educativas de la ciudad de Mendoza en Argentina, los docentes desarrollaron de la mejor manera las competencias relacionadas con el rol de la docencia. Ante el rápido e inesperado confinamiento social, los docentes procedieron a digitalizar el material didáctico y a la elaboración de las guías de estudio. Aunque no es objeto de este artículo, este estudio permite evidenciar que el uso de las clases online como recurso pedagógico es utilizado en su mayoría por docentes de instituciones privadas, lo que refleja la desigualdad de oportunidades educativas entre instituciones públicas y particulares.

Por su lado, en el estudio realizado en la institución de educación superior ubicada en el Valle del Cauca - Colombia, se distinguen las siguientes competencias: 1) Información y Alfabetización Internacional; 2) Comunicación y colaboración; 3) Creación del contenido digital; 4) Seguridad; 5) Resolución de problemas. Según los resultados de este estudio, las competencias más débiles desarrolladas están relacionadas con la creación de contenido digital y la seguridad. Aunque a muchos docentes les resulta relativamente fácil crear contenidos digitales, tienen problemas cuando tienen que editar material digital ya existente, o tienen dificultad en el manejo de las licencias (Creative Commons), lo que pone en evidencia la falta de cultura en el manejo de derechos del autor.

En lo que se refiere a la "seguridad", los docentes no manejan con mucha facilidad ciertos aspectos relacionados con la protección de datos personales, protección de identidad y protección digital. La competencia docente más desarrollada, según este estudio, es la "resolución de problemas". Los docentes pueden desarrollar competencias digitales relacionadas con las asignaturas que dictan, utilizando la creatividad tecnológica y generando recursos formativos que direccionen los conocimientos y habilidades de los estudiantes.

Por su lado, el estudio desarrollado en la Universidad Nacional Autónoma de México agruparon cuatro tipo de problemáticas a las que se están enfrentando los docentes para transformar sus actividades a la modalidad a distancia: 1) Logísticas; 2) Tecnológicas; 3) Pedagógicas; y 4) Socioafectivas. El 43,3\% de los docentes consideran que las problemáticas más frecuentes que tienen que enfrentar son las logísticas; las cuales están relacionadas con el manejo del tiempo, los horarios de clases, los espacios físicos para trabajar en esta modalidad, la comunicación institucional, entre otras. 
En segundo lugar, se encuentran las problemáticas tecnológicas, donde el $39,7 \%$ de los docentes coinciden que se torna dificultoso el acceso a internet, la disposición de equipos de cómputo, los conocimientos de plataformas educativas, entre otros. Un tercer grupo de docentes $(35,2 \%)$ coinciden con las problemáticas pedagógicas; es decir, no tienen suficiente conocimiento de herramientas didácticas de educación a distancia, el manejo de grupos a distancia, la evaluación de estudiantes, entre otros. Finalmente, un grupo reducido de docentes $(14,9 \%)$ enfrentan las problemáticas socioafectivas; es decir, aquellas que están relacionadas con aspectos emocionales, afectivos y de salud, sentimientos de tristeza, frustración, ansiedad, cansancio, entre otras.

A través de los resultados presentados en este artículo de revisión sistemática se puede concluir que se cumple con el objetivo planteado, ya que se ha podido evidenciar el desarrollo de las competencias digitales de los docentes universitarios en diferentes instituciones del continente americano. Sin embargo, será necesario elaborar un plan de fortalecimiento para las competencias docentes que no se han desarrollado satisfactoriamente, especialmente las relacionadas con el rol del docente (planificación, desarrollo, conducción de experiencias de aprendizaje presenciales con TIC), la creación del contenido digital, y la seguridad.

Si bien es cierto, los docentes son capaces de utilizar las tecnologías de la información en su vida personal y profesional, no es menos cierto que muchos de ellos carecen de innovación, factor considerado muy importante a la hora de enfrentar estos grandes retos como es la educación online en épocas de asilamiento preventivo producto de la pandemia mundial del COVID-19. Para ello, es inevitable estimular a los docentes universitarios a formar grupos virtuales para estudiar e investigar temas vitales para su profesión.

En la actual Sociedad del Conocimiento de la Información, las instituciones de educación superior les están asignando un rol protagónico a las competencias digitales; por lo que es necesario que las investigaciones de este tipo no se realicen en forma aislada sino que se articulen con otras universidades, y de esta manera se logre una visión integral y se pueda estudiar equitativamente esta problemática. 


\section{REFERENCIAS}

Amaya-Amaya, A., Zúñiga-Mieles, E., Salazar-Blanco, M., \& Ávila-Ramírez, A. (2018). Empoderar a los Profesores en su Quehacer Académico a través de Certificaciones Internacionales en Competencias Digitales. Apertura, 104-115.

Anaya Velasco, Y., Díaz Rodríguez, S., \& Martínez Hernández, J. (2012). El uso de las TIC como herramienta para el Aprendizaje Significativo del Inglés. Rastros Rostros, 14(27), 115-119.

Expósito, C. D., \& Marsollier, R. G. (2020). Virtualidad y Educación en Tiempos de COVID-19. Un estudio empírico en Argentina. Educación y Humanismo, 1-22.

Fernández Enguita, M. (2020). Obtenido de Una pandemia imprevisible ha traído la brecha previsible. : https://bit.ly/2VT3kzU

García-Peñalvo, F. J., Corell, A., Abella-García, V., \& Grande, M. (2020). La Evaluación Online en la Educación Superior en tiempos del COVID-19. Education in the Knowledge Society, 1-25.

Ilomäki, L., Kantosalo, A., \& Lakkala, M. (2017). What is digital competence? Retrieved from European Schoolnet: http://linked.eun.org/web/guest/in-depth3

Levano-Francia, L., Sánchez-Díaz, P., Guillén-Aparicio, P., Tello-Cabello, S., Herrera-Paico, N., \& Collantes-Inga, Z. (2019). Competencias Digitales y Educación. Propósitos y Representaciones, 569-588.

López Segrera, F. (2008). Tendencias de la Educación Superior en el Mundo y en América Latina y El Caribe. Revista da Avaliação da Educação Superior, 267-291.

Marco Europeo de Competencias Digitales para la Ciudadanía. (23 de Noviembre de 2017). Plan de Alfabetización Tecnológica. Obtenido de Somos Digital: https://somos-digital.org/disponibleonline-y-en-espanol-el-marco-europeo-de-competencias-digitales-para-la-ciudadania/

Martínez-Garcés, J., \& Garcés-Fuenmayor, J. (2020). Competencias Digitales Docentes y el Reto de la Educación Virtual derivado de la COVID-19. Educación y Humanismo, 1-16.

Moll, S. (2017). Marco Común de Competencia Digital Docente. España: Instituto Nacional de Tecnologías Educativas y Formación de Profesorado.

Organisation for Economic Co-operation and Development. (2005). The OECD Program Definition and Selection of Competencies. Retrieved from The definition and selection of key competencies.: http://www.oecd.org/

Pozos-Pérez, K. V., \& Tejada-Fernández, J. (2018). Competencias Digitales en Docentes de Educación Superior: Niveles de Dominio y Necesidades Fomrativas. Revista Digital de Investigación en Docencia Universitaria, 59-87.

Rangel Baca, A., \& Peñalosa Castro, E. A. (2013). Alfabetización Digital en Docentes de Educación Superior: Construcción y Prueba Empírica de un Instrmento de Evaluación. Píxel-Bit. Revista de Medios y Educación, 9-23.

Sánchez-Mendiola, M., Martínez-Hernández, A. M., Torres-Carrasco, R., De Agüero-Servín, M., Hernández-Romo, A. K., Beanvides-Lara, M. A., ... Jaimes-Vergara, C. A. (mayo-junio de 2020). Retos Educativos durante la Pandemia de COVID-19: Una encuesta a profesores de la UNAM. Revista Digital Universitaria, 21 (3), 1-24. 
Sandí-Delgado, J. C. (2020). Desarrollo de Competencias Digitales en el Profesorado a través de Juegos Serios: Un Estudio de Caso Aplicado en la Universidad de Costa Rica. E-Ciencias de la Información, 46-75.

Sandi-Delgado, J. C., \& Sanz, C. V. (2020). Jeugos Serios para Potenciar la Adquisición de Competencias Digitales en la Formación del Profesorado. Educación, 2215 - 2644.

Vuorikari , R. (2015, Mayo 21). Becoming digitally competent: A task for the 21st-century citizen. Retrieved from School Education Gateway: https://www.schooleducationgateway.eu/ en/pub/viewpoints/experts/riina_vuorikari__becoming_dig.htm

Zavala, D., Muñoz, K., \& Lozano, E. (2016). Un enfoque de las competencias digitales de los docentes. Publicando, $330-340$. 